\title{
La prise en charge globale des enfants en surpoids d'après le nouveau modèle suisse
}

Dagmar l'Allemand',

Nathalie Farpour-Lambert ${ }^{b}$, Bettina Isenschmid,

Josef Laimbacher ${ }^{a}$

a Hôpital des Enfants de l'Est de la Suisse, Saint-Gall

b Département de médecine communautaire, de premier recours et des urgences, Hôpitaux Universitaires de Genève

c Association Suisse Obésité de l'Enfant et de l'Adolescent (akj)

Traduction: Rudolf Schlaepfer, La Chaux-de-Fonds,

D. l'Allemand, N.J. Farpour-

Lambert

\footnotetext{
* Les références se trouvent sous www.bullmed.ch $\rightarrow$ Numéro actuel ou $\rightarrow$ Archives $\rightarrow 2014$ $\rightarrow 44$.
}

Correspondance:

Prof. Dr Dagmar l'Allemand Pädiatrische Endokrinologie/ Diabetologie Ostschweizer Kinderspital Claudiusstrasse 6

CH-9006 St. Gallen

Tél. 0712431467

Fax 0712437390
D'après les nouveaux résultats de l'EPF Zurich, le nombre d'enfants en surpoids et avec obésité n'a pas diminué. Il y a même des indices que l'activité physique et la masse musculaire métaboliquement active continuent de baisser chez les enfants en âge scolaire - et certainement pas seulement chez eux! Ce fait contribue à l'exacerbation des comorbidités. Ainsi les recommandations pour l'activité physique récemment publiées sous www.hepa.ch/internet/hepa/fr/ home/dokumentation/grundlagendokumente.html gagnent une importance majeure.

Bien qu'une tendance à la stabilisation du surpoids dans la population générale soit observée en Suisse, les groupes à haut risque restent difficiles à cibler, en particulier les enfants:

- qui ont des parents en surpoids,

- issus de l'immigration,

- de familles ayant un bas niveau socio-économique,

- qui ont des parents fumeurs,

- avec des problèmes psychiques, notamment le trouble du déficit de l'attention/hyperactivité (TDAH) $[1]^{*}$.

Sans traitement le surpoids constaté à l'âge de 2-6 ans persiste jusqu'à l'âge adulte [1]. Le médecin de famille (pédiatre, médecin généraliste-interniste) est en général la première personne qui prend en charge les enfants en surpoids et leurs familles. Cependant, celuici ne dispose souvent ni le temps ni les compétences nécessaires pour aborder les problèmes médicaux et psychosociaux complexes associés à l'obésité.

La nouvelle Ordonnance fédérale sur les prestations [2] permet enfin une prise en charge globale de l'obésité infantile, y compris les conseils diététiques. Les frais de thérapie multi-professionnelle structurée sont maintenant remboursés par les caisses-maladie en Suisse, tant pour les traitements multidisciplinaires structurés en individuel (MSIT) qu'en groupe (MGP), ceci avant qu'une comorbidité ne se manifeste. Des études nationales [3] et internationales [4] ont en effet mis en évidence que le traitement de l'obésité infantile est efficace et économique s'il respecte les principes suivants:

- Les critères pour le traitement d'obésité sont définis: tour de taille ou indice de masse corporelle (IMC) $>97$ e percentile (p.) ou >p.90 et avec la présence d'une comorbidité, ou rapport tour de taille/taille $>0.5$ ) [5].

- Les parents participent à la thérapie de l'enfant au besoin ils bénéficient d'une prise en charge

\section{Résumé}

A partir du 1.1.2014, chaque pédiatre et généraliste ainsi que médecin scolaire peut prendre en charge globale les enfants obèses au-dessous d'un IMC de $30 \mathrm{~kg} / \mathrm{m}^{2}$ : à côté des consultations médicales, des consultations diététiciennes et de physiothérapie peuvent être prescrites, avant qu'une comorbidité ne se manifeste. Si aucun des buts de thérapie n'est atteint après 6 mois et l'enfant et la famille sont motivés, un traitement en coopération multidisciplinaire d'un an au maximum est conseillé dans un cadre individuel ou en groupe, sous la conduite d'un médecin spécialisé pour l'obésité infantile. L'accréditation nécessaire est obtenue par une formation continue de 1 jour. Le suivi médical se poursuit pendant 2 à 5 ans.

personnelle afin d'aborder leur surpoids.

- Les patients sont motivés et les freins de la thérapie ont été identifiés et surmontés $\rightarrow$ (entretien motivationnel [6, 7])

- Le médecin collabore avec une équipe de thérapeutes des domaines de la psychologie, la diététique, et la physiothérapie ou de l'éducation physique adaptée.

- Ces professionnels ont suivi une formation continue spécifique pour le traitement d'enfants ou d'adolescents en surpoids [6].

- Les maladies psychiques et somatiques sousjacentes et les comorbidités ont été diagnostiquées et sont traitées de manière adéquate [8].

Nous proposons la manière de procéder suivante (fig. 1), les contenus ayant été décrits précédemment $[2,3,9]$ :

Prévention: Pour les enfants en surpoids (IMC $>$ p.90 et <p.97) sans comorbidité, il existe des programmes d'actions cantonaux (par ex. www.promo tionsante.ch), des camps [10] ou d'autres activités (www.akj-ch.ch/kinder-jugendliche/angebote.html).

- Phase I de la thérapie:

Lorsque les critères susmentionnés sont remplis, le médecin peut facturer ses propres consulta- 


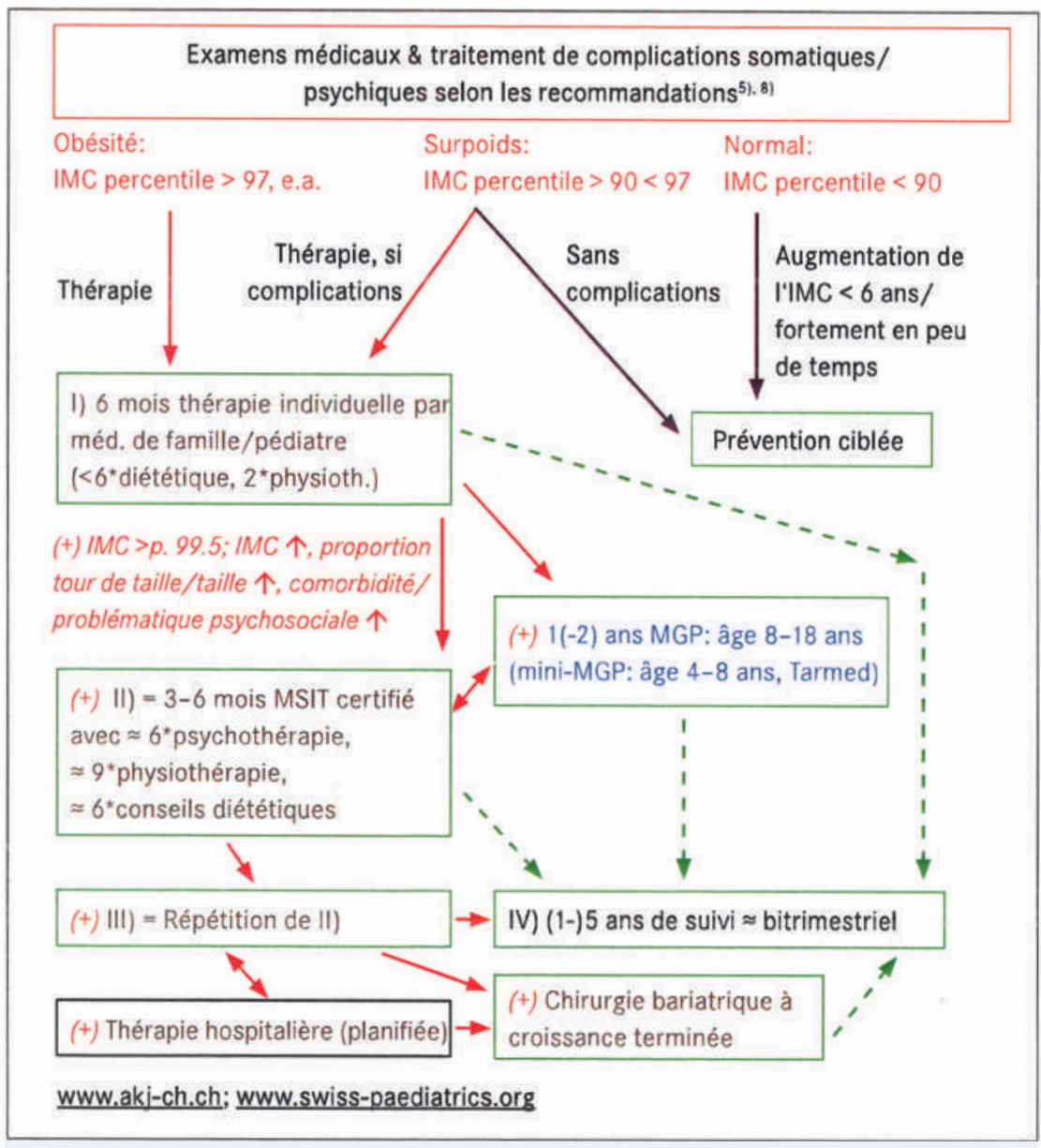

Figure 1

Modèle par étapes pour le traitement des enfants et adolescents avec surpoids en Suisse. Texte en rouge et (+): conditions; cadre vert: formes de traitement possibles depuis janvier 2014; $\approx$ : environ, valeur approximative; MGP: programmes de thérapie multi-professionnelle structurée de groupe; MSIT: programme de traitement multi-professionnel structuré individuel (cf. texte). est $>99.5^{\mathrm{e}}$ percentile, le patient peut immédiatement commencer la phase II.

- Phase III:

Dans une $3^{e}$ étape, un renouvellement est possible en cas d'échec de la phase II.

- Phase IV:

Le suivi médical se poursuit pendant 2 à 5 ans afin de prévenir une rechute, complété éventuellement par des mesures de prévention.

Il est important de surveiller les enfants dont il n'est pas possible de prendre en charge le surpoids, afin de garantir un dépistage et un traitement médicamenteux précoce des complications telle que l'hypertension, la dyslipidémie, la stéatohépatite non-alcoolique et le (pré-)diabète! Des comorbidités nécessitant un traitement sont présentes chez 12 à 25\% des enfants en surpoids, mais passent souvent inaperçues. En conséquence, moins de $1 \%$ des enfants sont adéquatement suivis et traités.

La Société Suisse de Pédiatrie (SSP) et l'Association Suisse Obésité de l'Enfant et de l'Adolescent (akj) sont responsables de la certification des MGP et de l'accréditation des médecins pour la thérapie individuelle multidisciplinaire. Tous les professionnels pratiquant déjà la thérapie multi-professionnelle de groupe sont systématiquement accrédités pour la thérapie individuelle [11]. Une formation continue d'une journée sur le traitement de l'obésité infantile est obligatoire pour pouvoir prescrire les étapes II-III du traitement individuel. Celle-ci est aussi vivement conseillée aux thérapeutes qui travaillent en réseau avec les médecins prescripteurs. La formation est mise en place par l'association spécialisée (l'akj, www.akj-ch.ch) ou par des institutions reconnues par l'akj et la SGP (par ex. programme de formation Contrepoids aux Hôpitaux Universitaires de Genève, http://contrepoids.hugge.ch, ou à l'Hôpital du Valais). La procédure de la certification sera publiée prochainement sur les sites Internet de la SSP et de l'akj. La liste des centres certifiés pour la thérapie de groupe et des médecins accrédités pour la thérapie individuelle sera mise à jour régulièrement par la SSP et l'akj. Sont également prévues des formations continues pour la prévention du surpoids et des troubles alimentaires au cabinet médical à l'intention des assistantes médicales.

Il existe depuis le 1.1.2014 l'obligation de prise en charge par les assurances-maladie des frais de traitement des programmes multidisciplinaires de groupe, mais l'ancien contrat tarifaire est arrivé à échéance. Il est conseillé de vérifier si l'ancien tarif de 4200 francs par enfant reste en vigueur, avant de commencer une thérapie de groupe. Des négociations sont actuellement en cours avec les différents assureurs pour adapter ce tarif aux coûts réels dans les groupes à 7-8 enfants. 


\section{Références}

1 l'Allemand D, Laimbacher J. Options and limits in the treatment of overweight children and adolescents and their families in primary care. Ther Umsch. 2013;70(11):695-702.

2 Ordonnance du DFI sur les prestations dans l'assurance obligatoire des soins en cas de maladie (Ordonnance sur les prestations de l'assurance des soins, OPAS), modification du 6 décembre 2013.

3 l'Allemand D, Kirchhoff E, Farpour-Lambert N et al. Evaluation du traitement des enfants et adolescents en surpoids en Suisse: Analyse intermédiaire KIDSSTEP de la thérapie dans les programmes de groupe multi-professionnels jusqu'au 1.5.2012. Paediatrica. 2012;23(5):27-30

4 Sargent GM, Pilotto LS, Baur LA. Components of primary care interventions to treat childhood overweight and obesity: a systematic review of effect. Obes Rev. 2011;12,e219-e235.

5 Jenni OG, Braegger C, Konrad D, Molinari L. Nouvelles courbes de croissance pour la Suisse. Paediatrica. 2011;22(1):9-11. www.swiss-paediatrics.org/sites/ default/files/empfehlungen/wachstumskurven/pdf/ perzentilen_2012_09_15_sgp_d.pdf
6 Berg-Smith SM, Stevens VJ, Brown KM et al. A brief motivational intervention to improve dietary adherence in adolescents. The Dietary Intervention Study in Children (DISC) Research Group. Health Educ Res. 1999;14:399-410.

7 KIDSSTEP-interview de motivation, www.akj-ch.ch

8 l'Allemand D, Farpour-Lambert N, Laimbacher J. Définition, diagnostic et indications thérapeutiques de la surcharge pondérale de l'enfant et de l'adolescent. Paediatrica 2006;17(6):19-24. www.swiss-paediatrics. org/sites/default/files/paediatrica/vol17/n6/pdf/ 13-28.pdf

9 Farpour-Lambert N, Sempach R, l'Allemand D, Laimbacher J. Thérapie de l'obésité de l'enfant et de l'adolescent: Propositions de programmes interdisciplinaires. Paediatrica. 2007;18(2):37-40. www.swiss-paediatrics.org/sites/default/files/ paediatrica/vol18/n2/pdf/37-40.pdf10 www.sportsmile.ch/fr/activities.php; www.crs-ne.ch www.asipao.ch

11 www.swiss-paediatrics.org/fr/informations/news/ therapie-contre-lobesite 